\title{
An investigation of solar flare effects on equatorial ionosphere and thermosphere using co-ordinated measurements
}

\author{
S. G. Sumod ${ }^{1,2^{*}}$ (i) and Tarun Kumar Pant ${ }^{1}$
}

\begin{abstract}
The response of equatorial ionosphere-thermosphere system to the X3.8 solar flare of January 17, 2005 has been studied using the coordinated measurements of GPS-derived Total Electron Content (TEC), Ol $630.0 \mathrm{~nm}$ dayglow and magnetic field measurements over a dip equatorial station Trivandrum $\left(8.5^{\circ} \mathrm{N}, 77^{\circ} \mathrm{E}\right.$, dip $\left.0.5^{\circ} \mathrm{N}\right)$, in India. It has been observed that Equatorial Electrojet (EEJ) as inferred using the ground-based magnetometers and GPS-derived TEC measurements show prompt enhancements during the peak flare, as expected. Interestingly, the temporal evolution of TEC at different latitudes revealed that the X3.8 class flare produced significant weakening of the plasma fountain and hence in the Equatorial Ionization Anomaly (EIA). Furthermore, the response of OI $630.0 \mathrm{~nm}$ dayglow during the flare is found to be strongly affected by the prevailing electrodynamics. The plausible physical mechanism for these effects is discussed in context of the current understanding of the neutral and electrodynamical coupling processes.
\end{abstract}

Keywords: Dayglow, Equatorial electrodynamics, Solar flare, Equatorial lonization Anomaly (EIA)

\section{Introduction}

Solar flares are sudden burst of radiations coming from the sun, which significantly alter various physical and chemical processes in the terrestrial upper atmosphere. Considerable number of studies have been made in the past, to understand the effects of solar flare over the ionospheric (e.g., Le et al. 2013 ) and thermospheric (e.g., Le et al. 2012, 2015; Pawlowski and Ridley 2008, 2011) altitudes. Multitude of data from ground/space-based platforms primarily using GPS TEC (Afraimovich 2000, Leonovich et al. 2002, 2010; Liu et al. 2006), incoherent scatter radars (Mendillo and Evans 1974) in tandem with theoretical modeling (Chamberlin et al. 2008; Qian et al. 2010) have provided significant results pertaining to the flare-induced effects over the terrestrial upper atmosphere.

Recently, solar flare effects and its repercussions on the electrodynamics of the equatorial ionosphere have got

\footnotetext{
*Correspondence: sgsumodh@gmail.com

1 Space Physics Laboratory, Indian Space Research Organization,

Trivandrum 695022, India

Full list of author information is available at the end of the article
}

significant attraction across the globe (e.g., Zhang et al. 2017; Manju 2016; Manju et al. 2012). Liu et al. (2007) has shown that Equatorial Ionization Anomaly (EIA) got significantly weakened during the flare due to the dominant role of electrodynamics than the photochemistry. Simulation studies (Qian et al. 2012) also showed that the $\boldsymbol{E} \times \boldsymbol{B}$ drift over equator weakens during the flare. This is consistent with the earlier observations of decrease in eastward electric field in the dynamo region during the flare (Manju and Viswanathan 2005). However, in past both increase (Qian et al. 2012) and decrease (Manju and Viswanathan 2005) in the field-line-integrated Hallto-Pedersen conductivity ratio over equator had been reported. On the other hand, the effects of solar flare on the neutral thermosphere, particularly over equatorial latitudes are not properly understood so far, mainly due to the paucity of observations. Although CHAllenging Mini satellite Payload (CHAMP) observations provided several new insights in this regard (Liu et al. 2007), they are normalized to a higher altitude of $400 \mathrm{~km}$ and have poor temporal resolution ( $\sim 93 \mathrm{~min}$.) due to the inherent time for the satellite to revisit the same latitude and local time. Therefore, the need for high cadence data 
from lower thermosphere is well appreciated for the comprehensive understanding of the ionospheric-thermospheric coupling during such transient events.

In this context, the ground-based neutral thermospheric OI $630.0 \mathrm{~nm}$ dayglow emissions are ideal for investigating such ephemeral events like flare, as they emanate from the altitudes, where maximum EUV absorption takes place. These dayglow measurements over Trivandrum, in recent years, have brought out many significant results pertaining to various aspects of coupling processes in the equatorial upper atmosphere (e.g., Sumod et al. 2011a, b, 2012, 2014, 2015 and references therein). In the present work, an attempt is made to investigate the effects of X3.8 solar flare, which occurred on January 17, 2005, over equatorial ionosphere-thermosphere system, using combined high cadence measurements of thermospheric OI $630.0 \mathrm{~nm}$ dayglow, GPS-measured ionospheric Total Electron Content (TEC) and the strength of Equatorial Electrojet (EEJ). The study perhaps provides the 'first time' experimental evidence for the weakening of EIA during the flare over the Indian longitudinal sector.

\section{Experiment}

The high cadence $(1 \mathrm{~min})$ data of thermospheric OI $630.0 \mathrm{~nm}$ dayglow have been obtained using a unique Multiwavelength Dayglow Photometer (MWDPM), over a magnetic dip equatorial station Trivandrum $\left(8.5^{\circ} \mathrm{N}, 77^{\circ}\right.$ E, dip $0.5^{\circ} \mathrm{N}$ ), in India. The comprehensive details of the system (Sridharan et al. 1998) and data analysis (Sumod et al. 2014) had been published elsewhere. For studying the $\mathrm{F}$ region response to the flares, the TEC measured using GPS receivers at different stations covering the trough to crest location of the EIA in the Indian longitudinal sector are used. The station names and their coordinates used are listed in Table 1. The flare effects in the dynamo region are studied using the 1-min values of the strength of EEJ (i.e., $\Delta H_{\mathrm{TVM}}-\Delta H_{\mathrm{ABG}}$ ) obtained using the magnetometer observations over an equatorial station Trivandrum (TVM) and an off-EEJ station Alibag

Table 1 List of the GPS receiver stations used along with their geographic co-ordinates

\begin{tabular}{lll}
\hline Station & Geographic lat. & Geographic long. \\
\hline Shimla & $31.1^{\circ} \mathrm{N}$ & $77.1^{\circ} \mathrm{E}$ \\
Delhi & $28.75^{\circ} \mathrm{N}$ & $77.25^{\circ} \mathrm{E}$ \\
Bhopal & $23^{\circ} \mathrm{N}$ & $77.2^{\circ} \mathrm{E}$ \\
Hyderabad & $17.5^{\circ} \mathrm{N}$ & $78.5^{\circ} \mathrm{E}$ \\
Bengaluru & $13^{\circ} \mathrm{N}$ & $77.5^{\circ} \mathrm{E}$ \\
Trivandrum & $8.5^{\circ} \mathrm{N}$ & $77^{\circ} \mathrm{E}$ \\
\hline
\end{tabular}

(ABG), where $\Delta H$ stands for the difference in the instantaneous values of horizontal component of magnetic field from its nighttime mean value. One-minute values of the X-ray (1-8 ̊̊) flux obtained using GOES-10 (Geostationary Operational Environmental Satellite) and 15 s EUV flux $(26-34 \mathrm{~nm}$ ) data obtained from the SEM (Solar EUV Monitor) onboard SOHO (Solar Heliophysical Observatory) are used, for characterizing the Solar flare event of January 17, 2005.

\section{Results and discussion}

\section{Solar flare event of January 17, 2005}

During the period 17-23 January 2005, solar activity varied from low to high levels. The period began under the influence of a high-speed coronal hole stream, with solar wind speed ranging between 550 and $600 \mathrm{~km} / \mathrm{s}$, later increasing up to $\sim 1000 \mathrm{~km} / \mathrm{s}$. Transient flow, likely associated with CME activity on 15 January, arrived at around 17 January at $\sim 07: 15 \mathrm{UT}$. The region 720 , which remained as the largest sunspot in the visible disk of the sun during this period, produced twenty five C-class, eight $\mathrm{M}$-class, and three $\mathrm{X}$-class flares. The first significant event was an X3.8/sf proton flare, occurred on January 17 at 09:52 UT and its effects over the equatorial upper atmosphere are studied here.

Figure 1 shows the time evolution of X-ray (1-8 $\AA ̊ ㇒)$ measured using GOES and EUV (26-34 nm) flux obtained from SOHO on 17 January 2005. As it is clear from the figure, an abrupt, but a small enhancement in X-ray followed by EUV occurred at $\sim 13: 25$ IST. Thereafter, both showed a steep enhancement at $\sim 15: 15$ IST reaching a maximum at 15:20 IST. It is long-duration flare $(\sim 4 \mathrm{~h})$, which continued till $\sim 17: 30$ IST. It is interesting to note that though the X-ray showed a single peak, double peaks have been observed in the EUV flux. In fact, a nonlinear relationship between the enhancements in X-ray and EUV during different solar flares had

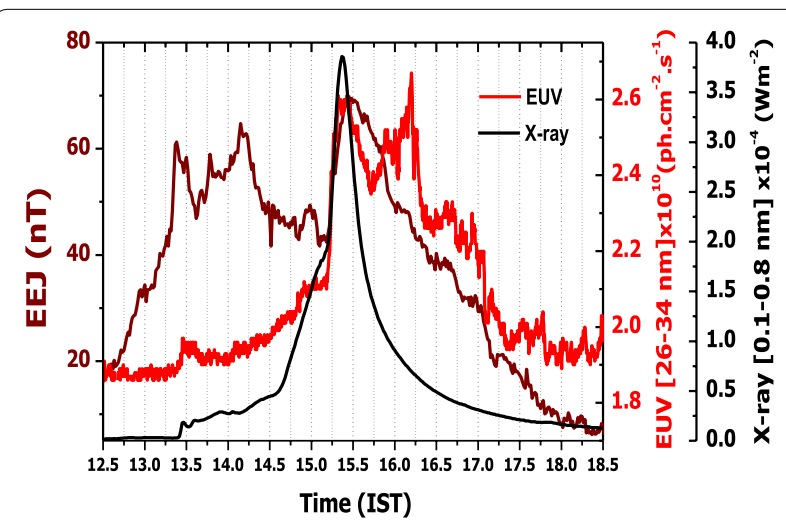

Fig. 1 The daytime variation of the X-ray and EUV flux along with EEJ 
also been reported in literature (Mahajan et al. 2010). The percentage enhancement in the EUV flux (difference in the EUV between the pre-flare and peak flare) is found to be $39 \%$. The following sections brief the effect of this X3.8 flare on the equatorial ionosphere followed by the equatorial thermosphere in context of thermosphereionosphere coupling.

\section{Response of equatorial/low latitude ionosphere Effects in the dynamo region}

The magnetic field measurements at an EEJ station Trivandrum and an off-EEJ station Alibag are used to investigate the solar flare effect in $\Delta H$, i.e., SFE $(H)$ in the dynamo region. The daytime variation of EEJ on January 17, 2005 during the period 12:30-18:30 IST is also shown in Fig. 1. As moderate geomagnetic activity was ongoing on this day, fluctuations due to solar wind/magnetospheric/high latitude origin are seen in the magnetic field measurements. However, at 13:15 IST, a step-like enhancement has been observed in the surface magnetic field measurements. This is prior to the increase in EUV/X-ray radiations and is associated with the sudden increase in the solar wind ram pressure (not shown here). However, in conjunction with the peak X-ray/EUV flux, a clear-cut enhancement is seen in the $\Delta H$ at Trivandrum, Alibag and hence in EEJ. The SFE in $\Delta H\left(\Delta H_{\text {peak }}\right.$ flare $\left.-\Delta H_{\text {pre flare }}\right)$ over Trivandrum and Alibag is $\sim 28$ and $\sim 2 \mathrm{nT}$, respectively. Therefore, the flare-associated enhancement in EEJ was conspicuous having magnitude of $\sim 26 \mathrm{nT}$. This shows that the SFE in $H$ decreased drastically from equator to low latitudes.

Recent studies showed that the zonal electric field and, hence, the EIA get significantly weakened during the X-class flare event of 28 October 2003 (Liu et al. 2007). To investigate this aspect in detail, the GPS-measured $\mathrm{TEC}$ at six different stations in the $\sim 77^{\circ} \mathrm{E}$ meridian have been analyzed and the results are presented below.

\section{Changes as seen in the TEC}

As it is well known, GPS satellites and dual-frequency receivers provide very high cadence (1 $\mathrm{min}$. and less) TEC measurements. These GPS-measured TEC data are ideal for investigating the solar flare effects, as the transmitted frequencies $L 1$ and $L 2$ are higher than the ionospheric collision frequencies, and minimize the signal fade out. (e.g., Manju et al. 2009). The pseudoranges and carrier phase delays of $L 1$ and $L 2$ are used to derive the absolute Vertical TEC (VTEC) measurements. Figure 2 shows the temporal evolution of rate of change of TEC (rTEC) along with the absolute VTEC and X-ray/EUV flux variations during the period $12: 30-18: 30$ IST for the station Bengaluru $\left(13^{\circ} \mathrm{N}, 77.5^{\circ}\right.$ E), obtained using a single satellite (PRN). A clear-cut

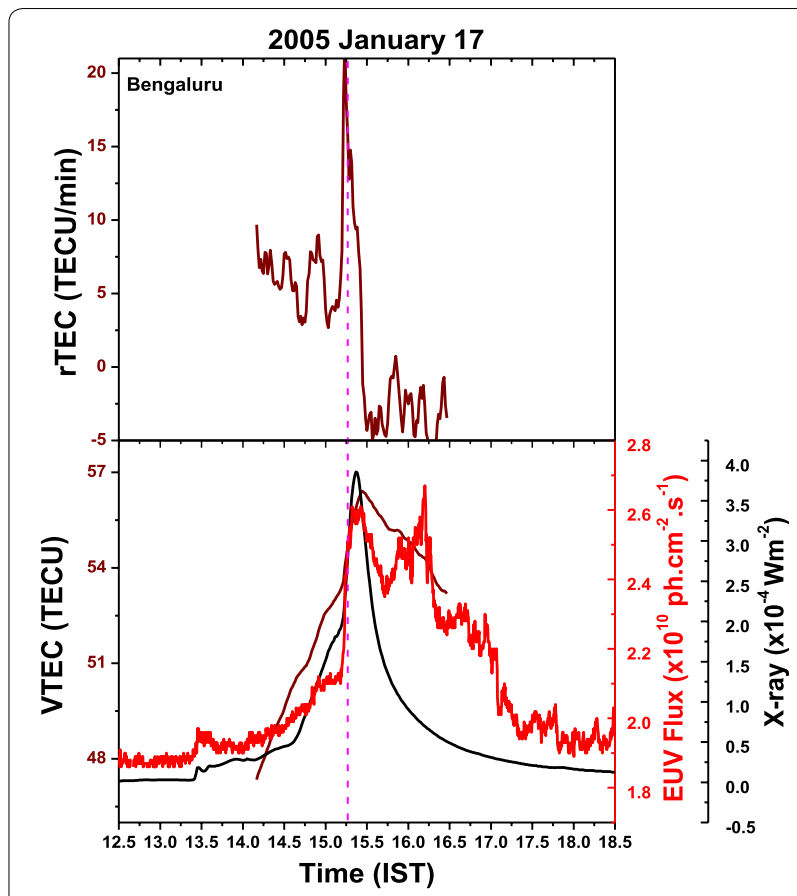

Fig. 2 Time evolution of VTEC variations obtained for a particular PRN at Bengaluru on January 17, 2005 along with the X-ray and EUV flux (bottom panel). Top panel shows the rate of change of TEC. The dotted line (magenta) indicates the peak in the rTEC

enhancement in both TEC and rTEC in consonance with flare radiations is evident from the figure. Similar enhancements were also noticed from all the five other stations, used in this study. It is to be noted that here only those raypaths with elevation angles greater than $50^{\circ}$ are used, as it has been reported that they are ideal for representing TEC over Indian region (Rama Rao et al. 2006).

To address the effect of flare-induced changes in the time evolution of EIA, the latitudinal distribution of VTEC at every $30 \mathrm{~min}$ along the $\sim 77^{\circ} \mathrm{E}$ during the period 12:00-17:00 IST is shown in Fig. 3 (top panels). For comparison, the same for the control day (13th February 2005) is also shown in the bottom panels. For obtaining the latitudinal profiles of VTEC, data from all the six stations have been used. As we have used the GPS TEC data with elevation cut off greater than $50^{\circ}$, we can have more latitudinal coverage from a single station. The VTEC values obtained from different stations along the $\sim 77^{\circ} \mathrm{E}$ longitude listed in Table 1 are interpolated to obtain these latitudinal profiles. In fact, similar kind of profiles has been successfully used elsewhere (Sumod et al. 2014, Sreeja et al. 2009 and references therein), for showing the temporal evolution of EIA. The same methodology is adopted here also, for obtaining the latitudinal variation of TEC during the flare time. It is to be noted that, here, we have 


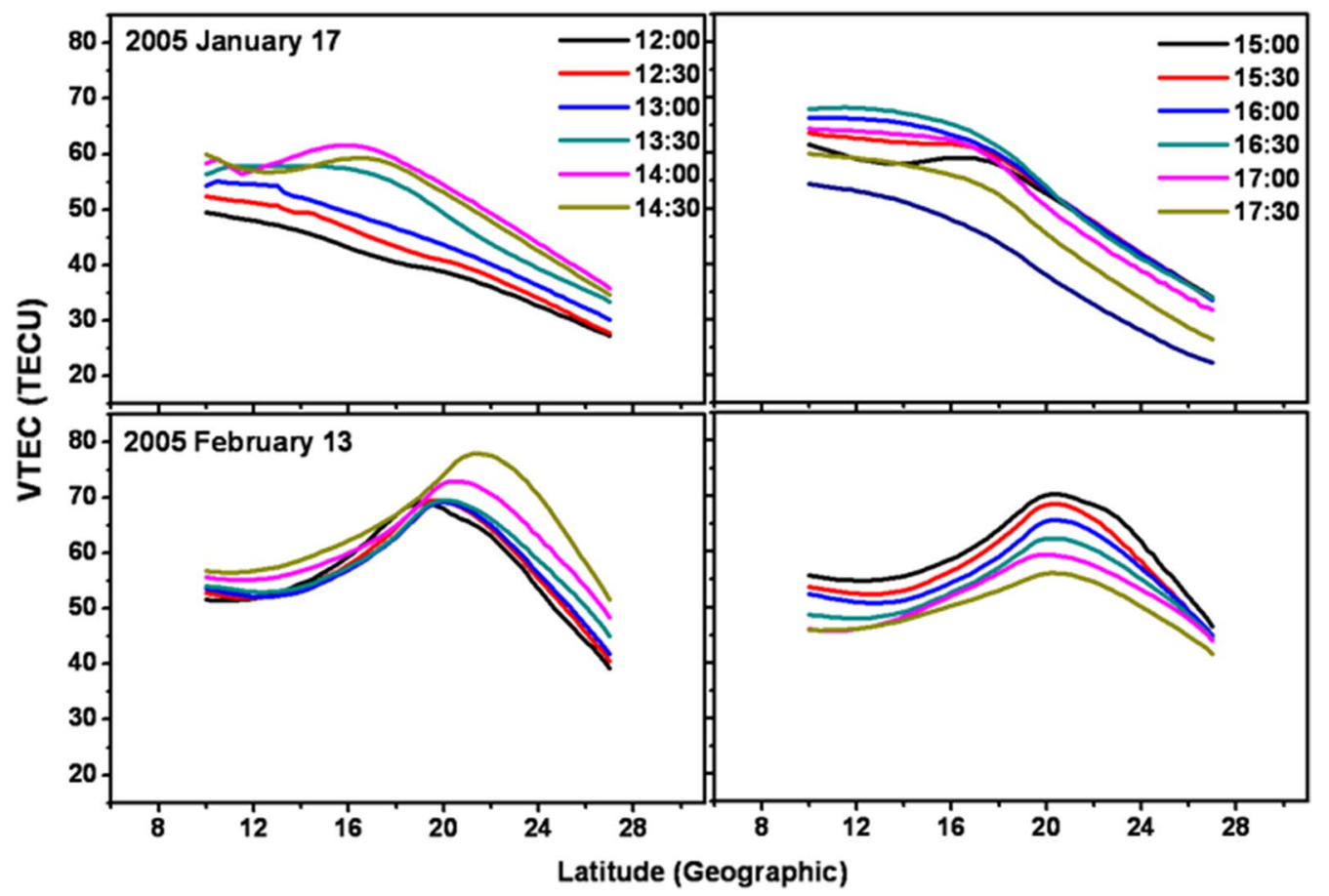

Fig. 3 Latitudinal variation of VTEC during the period 12:00-17:30 IST on January 17, 2005 (top panels) in comparison with the same for control day The peak flare time is 15:20 IST

zoomed the data from $10^{\circ}$ to $27^{\circ}$, to show the reduction in the vertical drift, if any, during flare duration more clearly. As mentioned earlier, a clear-cut development of anomaly has been observed on the control day. However, the evolution of the EIA, as seen in the TEC, on January 17, 2005 is found to be quite different. On this day, the anomaly development is found to be inhibited prior to the flare, suggesting that the relevant electrodynamical processes on this day had been rather weak or less intense.

However, the latitudinal distribution of VTEC at $\sim$ 13:30 IST showed a gradual increase over the latitudes $12-20^{\circ}$, indicative of the development of the EIA. The subsequent profiles during the period 14:00-15:00 IST exhibited gradual growth of EIA, with the crest location shifting systematically from latitudes $15^{\circ}$ to $17^{\circ}$. Although, the flare-associated enhancement in the VTEC throughout the latitudes can be clearly seen on the profiles from 13:30 IST onwards, the additional increase in VTEC within $12-20^{\circ}$ as compared with other latitudes clearly indicates the role of EIA-associated electrodynamics. From 15:30 IST onwards, the anomaly again showed a significant weakening, which continued until 17:30 IST. Therefore during the flare period (flare peak time is 15:20 IST), inhibition of the EIA has been observed. These results are consistent with the recent observations using the CHAMP, where a significant weakening of the
EIA-associated plasma fountain has been reported during the X17 flare event on 28th October 2003 (Liu et al. 2007).

To understand the causative mechanism for the observed temporal variability in the EIA discussed above, the time variations of EEJ on both the flare and control days are shown in Fig. 4. As it is clear from the figure, a

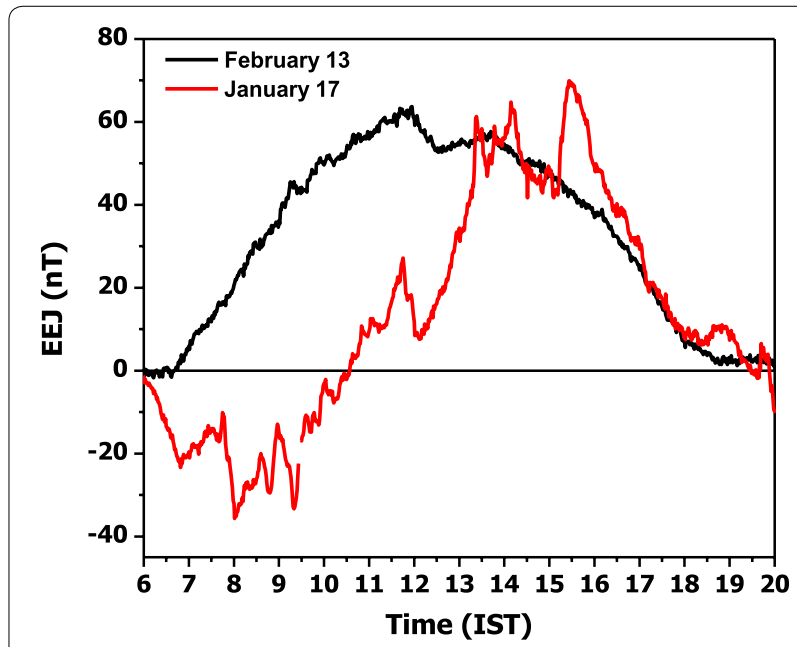

Fig. 4 The time variation of EEJ on January 17, 2005 as compared with the control day 
negative excursion has been observed in the EEJ on the flare day, till $\sim 11: 00$ IST. A substantial decrease in the EEJ till 13:00 IST can be noticed on the flare day in comparison with that on the control day.

In an earlier study, it has been shown that on the flare day, the development of anomaly was inhibited till 13:00 IST owing to the effect of the disturbance dynamo (Sreeja et al. 2009). Therefore, the weakening of the anomaly during the pre-flare period (12:00-13:00 IST) can be attributed mainly to the westward electric field associated with disturbance dynamo. However, the development of anomaly crest during the period 13:30-15:00 IST necessitates the presence of an eastward electric field. The positive excursion of EEJ from 13:00 IST as well as the solar wind pressure-induced increase in the EEJ at $\sim 13: 30$ IST vindicates the presence of an eastward electric field. This eastward electric field enhances the plasma fountain and hence the anomaly after certain period, as there involves a characteristic delay of $\sim 30-90$ min between EEJ and EIA.

However, after the pressure-induced enhancement at $\sim$ 13:20 IST, the EEJ followed in a similar pattern as that of the control day with an additional enhancement due to the flare-induced radiations. This suggests that from 15:30 IST onwards, the EIA should either grow in a similar pattern as that of the control day or develop further due to the additional positive amplitudes seen in the EEJ. Interestingly, contradictory to the expected behavior, it is found that the EIA got completely suppressed from 15:30 IST onwards which continued the rest of the day. This strongly corroborates the flare-associated weakening of the EIA as reported in the recent study (Liu et al. 2007). This weakening of EIA is believed to be associated with the reduction in the eastward electric field due to the flare. As it is well known, the flare is expected to cause the rapid change in the conductivity, especially in the vertical direction. This in turn affects the ratio of Hall to Pedersen conductivity, which is a crucial factor in regulating the dynamo electric field. As the present case is an X3.8 class flare, it can increase the conductivity (particularly below $100 \mathrm{~km}$ ), due to the increase in ionization at $\mathrm{D}$ region by the $\mathrm{X}$-rays. This in turn can decrease the zonal electric field depending on the vertical polarization electric field and ratio of the Hall to Pedersen conductivity. Similarly, the height-varying winds in the vicinity of the dynamo region due to the formation of a highly conducting layer can also modulate the zonal electric field (Liu et al. 2007). Therefore, the flare-induced changes in the EIA can be attributed to the combined effect of the photochemistry and the electrodynamics related to the plasma fountain.

As geomagnetic activities were ongoing during the period, variations of solar wind speed $(\mathrm{Vx})$, interplanetary magnetic field (IMF $\mathrm{Bz}$ ) and high-resolution Dst index (Sym-H), as obtained using the OMNI web (http://omniweb.gsfc.nasa.gov/), were plotted along with flare radiations in Fig. 5 below. As it is obvious from the figure, none of the parameters show concurrent variation with either X-ray or EUV radiation during the flare. It is to be noted that flare radiations slowly increased from $\sim$ 13:30 IST onwards, reaching its peak at $\sim 15: 20$ IST and came back to its pre-flare value at $\sim 18: 00$ IST. However, sharp rise in the X-ray flux/EUV has been noticed during 15:10-15:35 IST. The latitudinal profile at 15:00 IST showed a moderately developed EIA; whereas, profiles during 15:30-17:30 IST showed significant weakening. This concludes that after the peak flare, EIA has weakened substantially. As there is no southward/northward excursion of IMF Bz, during the period 15:0017:00 IST, the equatorial electric field is not expected to change during this period either due to overshielding or undershielding effect. Furthermore, sym- $\mathrm{H}$ remained more or less in a steady level in the range $20-40 \mathrm{nT}$ during this period, with a nearly constant solar wind velocity $\sim 625 \mathrm{~km} / \mathrm{s}$. Therefore, one cannot expect a changeover in the direction of equatorial electric field owing to the storm or penetration effects during this time. Although the solar and geomagnetic activities are not extremely

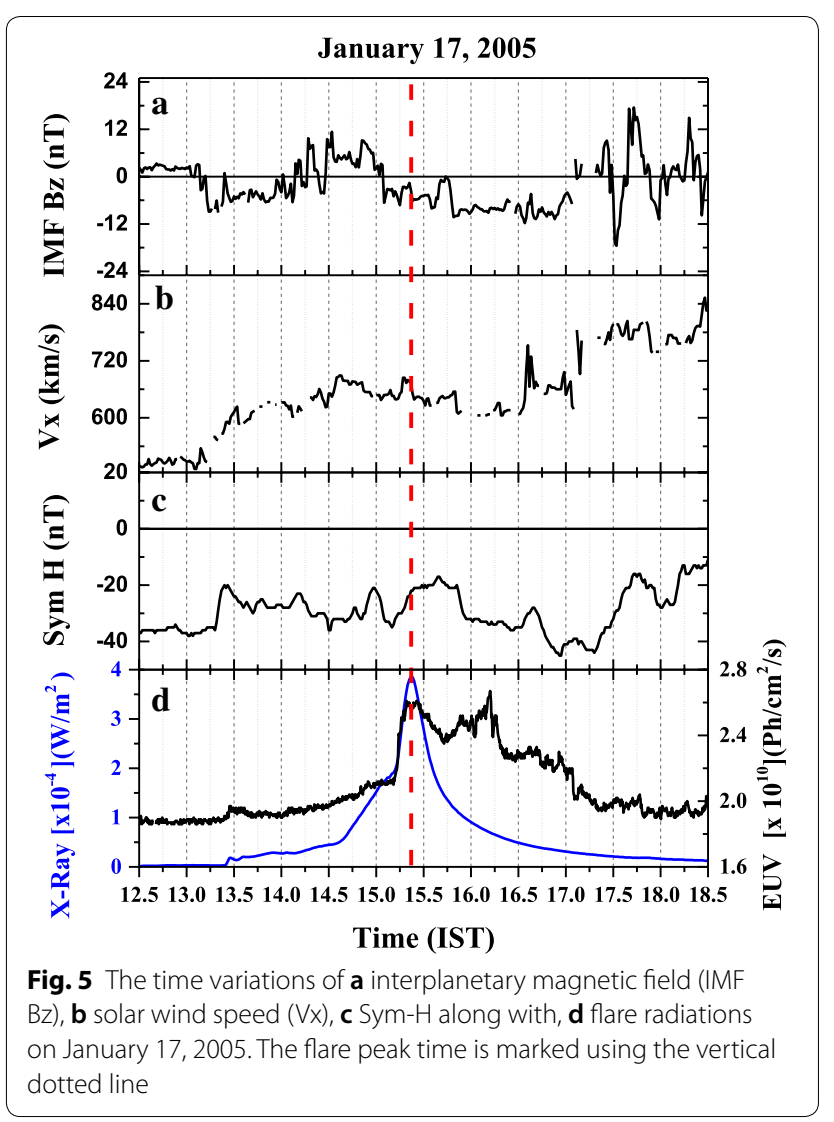


quiet, the absence of any significant or abrupt variations in the afore-mentioned parameters during/prior to the flare period strongly supports the conclusions drawn in the paper. Therefore, we believe that the observed variability in EEJ /EIA in consonance with X-ray and EUV is primarily due to the flare-induced radiations.

\section{Response of equatorial thermosphere}

To further investigate the thermospheric response of the X3.8 flare on January 17, 2005, the time variation of the OI $630.0 \mathrm{~nm}$ dayglow on this day during the period 12:00-18:00 IST is plotted in Fig. 6 (bottom panel). For comparison, the same on a control day (January 25, 2005) is also plotted in the same panel. As it is clear from the Figure, three distinct signatures (highlighted using circles) on January 17, 2005 can be observed during this period as compared with the normal day's behavior. As there were significant variabilities in both EEJ and flare radiations during this time, these signatures are attributable to the combination of the flare-induced changes and electrodynamics as described below.

As mentioned earlier, the eastward electric field increased at $\sim 13: 20$ IST due to the increase in the solar wind dynamic pressure. The associated development of EIA as shown in Fig. 3 further confirms this aspect. This results in the pumping of more ionization over the equator due to the upward $\boldsymbol{E} \times \boldsymbol{B}$ drift. This increases the number of effective dissociative recombination at the emission altitudes due to the pumping of enhanced ionization in the emission region. This in turn increases the airglow intensity over Trivandrum as seen in the present case. Therefore, the increase in airglow intensity, a few minutes after the increase in the EEJ at 13:20 IST, can be attributed to the prevailing eastward electric field.

This increase in dayglow continued until 14:30 IST, and showed an abrupt decrease at $\sim 14: 45$ IST, which persisted until $\sim$ 15:00 IST. The reduction in the dayglow intensity during the period 14:30-15:00 IST can be attributed to the decrease in the effective recombination at the emission altitudes due to the transport of more ionization density from equator to off-equatorial latitudes because of the prevailing eastward electric field. When the eastward electric field becomes strong enough to pump the ionization density from equator to off-equatorial latitudes, it would appear as a decrease in the dayglow over equator. The strengthening of EIA profiles, as shown in Fig. 3, further vindicates the presence of strong eastward electric field during this period. Moreover, a gradual decreasing trend in the dayglow is expected in the post-noon sector due the solar zenith angle dependence of photoelectron impact of $\mathrm{O}$ and photo-dissociation of $\mathrm{O}_{2}$, which contributes to the production of $\sim 70 \%$ of the dayglow intensity. Following this reduction, the dayglow intensity increased

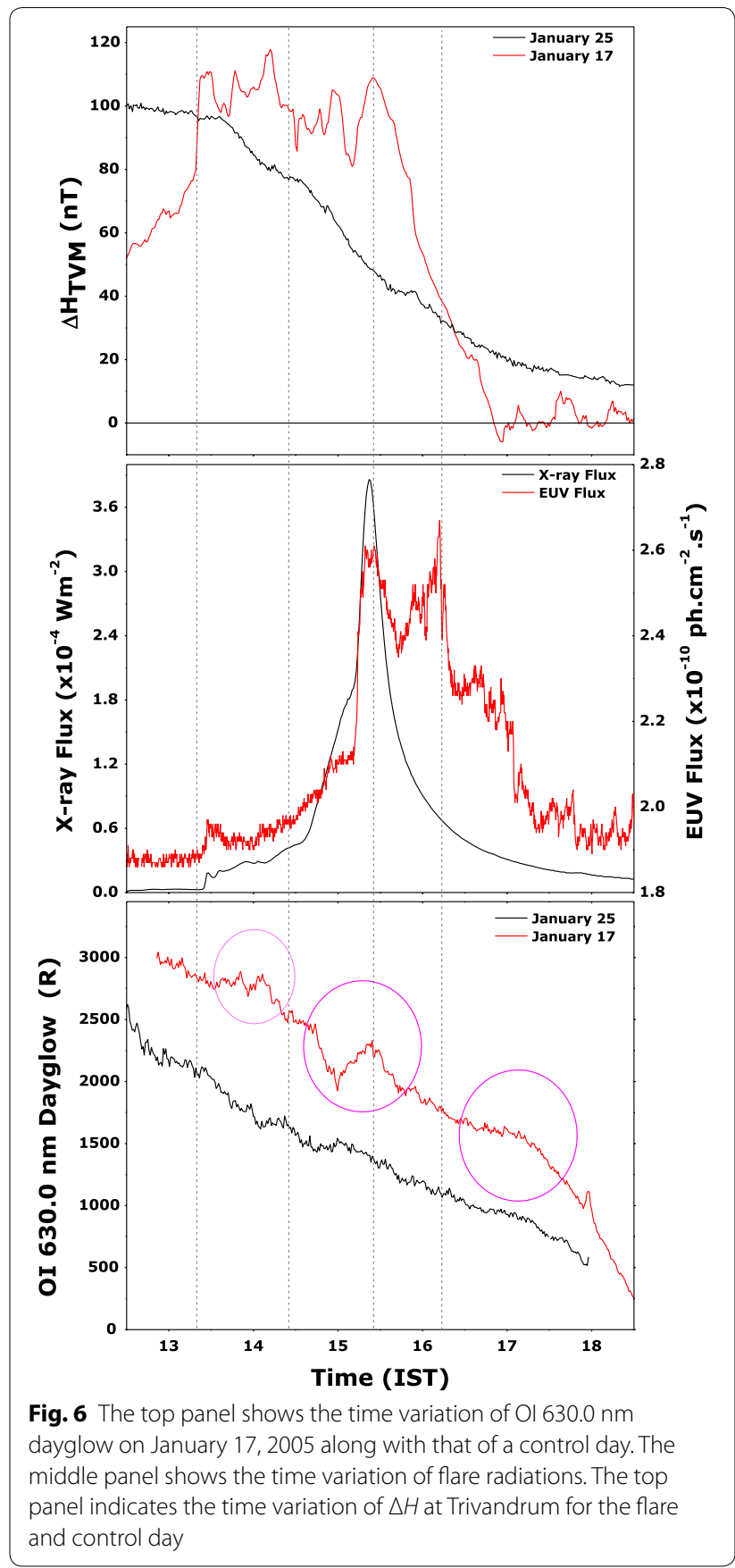

concomitantly with the X-ray/EUV radiations during the flare event. This increase in dayglow, which showed a peak at $\sim 15: 30$ IST, is attributed to the increased $\mathrm{O}\left({ }^{1} \mathrm{D}\right)$ production during the flare time due to all the three production mechanisms, viz, photo-electron impact of $\mathrm{O}$, photo-dissociation of $\mathrm{O}_{2}$ and dissociative recombination of $\mathrm{O}_{2}{ }^{+}$, as discussed in Sumod et al. (2015).

However, the dayglow did not show any appreciable signature associated with the secondary peak in the EUV flux. This is due to the fact that, during this period, the 
EEJ started to recover and flare-associated westward electric field, as discussed early, started dominating. This weakened the plasma fountain over equator, and filled more ionization over equator. This further increased the ionization at the emission altitudes, resulting in an increase in the dayglow intensity. Thus, the electrodynamics-associated plasma dominated over equator, diminishing the flare-induced peak in the dayglow intensity. The further enhancement in the dayglow intensity during the period 16:30-17:30 IST can be attributed to the subsequent weakening of plasma fountain as it is evident from Fig. 3. However, the delayed enhancement in the dayglow due to the time delay in the charge exchange of $\mathrm{O}_{2}+\mathrm{O}^{+}$reaction, as well as the delayed response of neutral density during the flare, cannot be precluded in this context.

\section{Conclusion}

The study adduces the effects of X3.8 flare of January 17, 2005 over the equatorial upper atmosphere using combined radio and optical measurements. Prompt responses have been noticed in the magnetic field inferred EEJ and GPS-measured TEC measurements. The latitudinal distribution of TEC revealed a substantial reduction in the EIA. This is consistent with the earlier observations/ simulations, and suggests that the flare-induced radiation has altered the ratio of Hall to Pedersen conductivity and subsequently resulted in the weakening of the eastward electric field. Although, the thermospheric dayglow revealed concomitant resolve enhancement during the peak flare period, it did not show any enhancement associated with later increase in the flare (EUV) radiation. This suggests the dominant role of electrodynamical imprints of these dayglow features when compared with the photochemistry. The importance of the study lies in showing the flare-induced changes in equatorial electrodynamics comprehensively in context of ion-neutral coupling, using high cadence coordinated ionospheric and thermospheric measurements.

\section{Acknowledgements \\ SGS acknowledges C. Vineeth and M.M. Hossain for the useful discussions and data collection. This work was supported by Department of Space, Govern- ment of India. SGS also acknowledges the financial assistance provided by the Indian Space Research Organization (ISRO) through research fellowship. Authors are thankful to the Director, IIG for the magnetic field data. The GOES \& SOHO team are also duly acknowledged.}

\section{Authors' contributions}

SGS conceived and designed the present study, performed the experiment in addition to collection of other associated data, carried out the data analysis, drafted the manuscript, and coordinated the study. TKP participated in designing the study, data analysis, as well as in the useful discussions concerning the study. Both authors read and approved the final manuscript.

\section{Funding}

This work was supported by Department of Space, Government of India through the research associateship to SGS (2013).

\section{Availability of data and materials}

The GOES data are available from http://goes.gsfc.nasa.gov/Data/goes.html and SOHO data from www.usc.edu/dept/space_science/semdata.html. The dayglow and GPS data are the property of ISRO and can be accessed at wWW. spl.gov.in. The magnetic field data are available at http://www.wdciig.res.in.

\section{Competing interests}

The authors declare that they have no competing interests.

\section{Author details}

1 Space Physics Laboratory, Indian Space Research Organization, Trivandrum 695022, India. ${ }^{2}$ Space Science Group, Sacred Heart College, Mahatma Gandhi University, Kochi 682 013, India.

Received: 30 January 2019 Accepted: 4 November 2019

Published online: 21 November 2019

\section{References}

Afraimovich EL (2000) GPS global detection of the ionospheric response to solar flares. Radio Sci 35(6):1417-1424. https://doi.org/10.1029/2000R S002340

Chamberlin PC, Woods TN, Eparvier FG (2008) Flare irradiance spectral model (FISM): flare component algorithms and results. Space Weather 6:S05001. https://doi.org/10.1029/2007sw000372

Le H, Liu L, Wan W (2012) An analysis of thermospheric density response to solar flares during 2001-2006. J Geophys Res 117:A03307. https://doi. org/10.1029/2011 JA017214

Le H, Liu L, Chen Y, Wan W (2013) Statistical analysis of ionospheric responses to solar flares in the solar cycle 23. J Geophys Res Space Phys 118:576582. https://doi.org/10.1029/2012JA017934

Le H, Ren Z, Liu L, Chen Y, Zhang H (2015) Global thermospheric disturbances induced by a solar flare: a modeling study. Earth Planets Space 67:3. https ://doi.org/10.1186/s40623-014-0166-y

Leonovich LA, Afraimovich EL, Romanova EB, Taschilin AV (2002) Estimating the contribution from different ionospheric regions to the TEC response to the solar flares using data from the international GPS network. Ann Geophys 20:1935-1941

Leonovich LA, Tashchilin AV, Portnyagina OYu (2010) Dependence of the ionospheric response on the solar flare parameters based on the theoretical modeling and GPS data. Geomagn Aeron 50(2):201-210. https://doi. org/10.1134/S0016793210020076

Liu JY, Lin CH, Chen Yl, Lin YC, Fang TW, Chen CH, Chen YC, Hwang JJ (2006) Solar flare signatures of the ionospheric GPS total electron content. J Geophys Res 111:A05308. https://doi.org/10.1029/2005JA011306

Liu H, Lühr H, Watanabe S, Köhler W, Manoj C (2007) Contrasting behavior of the thermosphere and ionosphere in response to 28 October, 2003 solar flare. J Geophys Res 112:A07305. https://doi.org/10.1029/2007JA012313

Mahajan KK, Lodhi NK, Upadhayaya AK (2010) Observations of X-ray and EUV fluxes during $X$-class solar flares and response of upper ionosphere. J Geophys Res 115:A12330. https://doi.org/10.1029/2010JA015576

Manju G (2016) On the unique divergent response of the equatorial electrojet vertical polarization electric field to different solar flares. Ann Geophys 27:3853-3860

Manju G, Viswanathan KS (2005) Response of the equatorial electrojet to solar flare related X-ray flux enhancements. Earth Planets Space 57:231-242. https://doi.org/10.1186/BF03351819

Manju G, Simi KG, Nayar SR (2012) Analysis of solar EUV and X-ray flux enhancements during intense solar flare events and the concomitant response of equatorial and low latitude upper atmosphere. J Atmos Sol Terr Phys 86:1-5

Manju G, Pant TK, Devasia CV, Ravindran S, Sridharan R (2009) Electrodynamical response of the Indian low-mid latitude ionosphere to the very large solar flare of 28 October 2003 - a case study. Ann Geophys 27:3853-3860

Mendillo M, Evans JV (1974) Incoherent scatter observations of the ionospheric response to a large solar flare. Radio Sci 9:197-210

Pawlowski DJ, Ridley AJ (2008) Modeling the thermospheric response to solar flares. J Geophys Res 113:A10309. https://doi.org/10.1029/2008JA013182 
Pawlowski DJ, Ridley AJ (2011) The effects of different solar flare characteristics on the global thermosphere. J Atmos Sol Terr Phys 73:1840-1848. https:// doi.org/10.1016/j.jastp.2011.04.004

Qian L, Burns AG, Chamberlin PC, Solomon SC (2010) Flare location on the solar disk: modeling the thermosphere and ionosphere response. J Geophys Res 115:A09311. https://doi.org/10.1029/2009JA015225

Qian L, Burns AG, Liu H, Chamberlin PC (2012) Solar flare impacts on ionospheric electrodynamics. Geophys Res Lett 39:L06101. https://doi. org/10.1029/2012GL051102

Rama Rao PVS, Gopi Krishna S, Niranjan K, Prasad DSVVD (2006) Temporal and spatial variations in TEC using simultaneous measurements from the Indian GPS network of receivers during the low solar activity period of 2004-2005. Ann Geophys 24:3279-3292

Sreeja V, Ravindran S, Pant TK, Devasia CV, Paxton L (2009) Equatorial and low latitude lonosphere-thermosphere system response to the space weather event of August 2005. J Geophys Res 114:A12307

Sridharan R, Modi NK, Pallam Raju D, Narayanan R, Pant TK, Taori A, Chakrabarty D (1998) A Multiwavelength daytime photometer-a new tool for the investigation of atmospheric processes. Meas Sci Technol 9:585-591

Sumod SG, Pant TK, Vineeth C, Hossain MM (2011a) A new insight into the vertical neutral-ion coupling between the mesopause and ionosphere $F$ region. Ann Geophys 29:421-426
Sumod SG, Pant TK, Vineeth C, Hossain MM, Antonita M (2011b) Response of tropical mesopause to the longest annular solar eclipse of this millennium. J Geophys Res 116:A06317. https://doi.org/10.1029/2010JA016326)

Sumod SG, Pant TK, Jose L, Hossain MM, Kumar KK (2012) Signatures of sudden stratospheric warming on equatorial thermosphere-ionosphere system. Planet Space Sci 63-64:49-55. https://doi.org/10.1016/j.pss.2011.08.005

Sumod SG, Pant TK, Vineeth C, Hossain MM (2014) On the ionospheric and thermospheric response of solar flare events of 19 January 2005: an investigation using radio and optical techniques. J Geophys Res 119:5049-5059

Sumod SG, Pant TK, Vineeth C, Hossain MM (2015) Unusual depletion of Ol $630.0 \mathrm{~nm}$ dayglow and simultaneous heating during the penetration of interplanetary electric field over dip equator. J Geophys Res 120:2110-2117

Zhang R, Liu L, Le H, Chen Y (2017) Equatorial ionospheric electrodynamics during solar flares. Geophys Res Lett. https://doi.org/10.1002/2017gl0733 238

\section{Publisher's Note}

Springer Nature remains neutral with regard to jurisdictional claims in published maps and institutional affiliations.

\section{Submit your manuscript to a SpringerOpen ${ }^{\circ}$ journal and benefit from:}

- Convenient online submission

- Rigorous peer review

- Open access: articles freely available online

- High visibility within the field

- Retaining the copyright to your article

Submit your next manuscript at $\boldsymbol{\nabla}$ springeropen.com 